\title{
Free electron laser and microwave instability interplay in a storage ring
}

\author{
G. L. Orlandi, ${ }^{*}$ C. Bruni, D. Garzella, and M. E. Couprie \\ CEA/DSM/DRECAM/SPAM, bâtiment 522, 91191 Gif-sur-Yvette, France \\ and LURE, Université Paris-Sud, bâtiment 209D, BP 3491898 Orsay, France \\ C. Thomas \\ LURE, Université Paris-Sud, bâtiment 209D, BP 3491898 Orsay, France \\ and Technische Universteit Eindhoven, Eindhoven, The Netherlands \\ R. Bartolini, C. Rippon, and G. Dattoli \\ ENEA, Divisione Fisica Applicata, Centro Ricerche Frascati, Roma, Italy \\ (Received 22 September 2003; published 2 June 2004)
}

\begin{abstract}
Collective effects, such as the microwave instability, influence the longitudinal dynamics of an electron beam in a storage ring. In a storage ring free electron laser (FEL) they can compete with the induced beam heating and thus be treated as a further concomitant perturbing source of the beam dynamics. Bunch length and energy spread measurements, carried out at the Super-ACO storage ring, can be correctly interpreted according to a broad-band impedance model. Quantitative estimations of the relative role that is played by the microwave instability and the laser heating in shaping the beam longitudinal dynamics have been obtained by the analysis of the equilibrium laser power. It has been performed in terms of either a theoretical limit, implemented with the measured beam longitudinal characteristics, or the numerical results obtained by a macroparticle tracking code, which includes the laser pulse propagation. Such an analysis, carried out for different operating points of the Super-ACO storage ring FEL, indicates that the laser heating counteracts the microwave instability.
\end{abstract}

DOI: 10.1103/PhysRevSTAB.7.060701

PACS numbers: 41.60.Cr, 29.20.Dh, 29.27.Bd

\section{INTRODUCTION}

Free electron lasers (FEL) are tunable coherent sources of radiation with a spectral range extending from the infrared to the vacuum ultraviolet (VUV). The coherent radiation emission results from the interaction between a relativistic microbunched charged beam crossing the periodic magnetic field of an undulator and a light pulse. In FEL oscillators, the laser oscillation is originated by the interaction between a charged bunch passing through the FEL straight section and a light pulse that is confined in an optical cavity, provided that the perfect synchronism condition is achieved. The energy of the relativistic charged beam defines the radiation spectral range, whereas the wavelength can be tuned by varying either the beam energy or the undulator magnetic field. The laser amplification mechanism is produced to the detriment of the kinetic energy of the charged beam: a fraction of it is converted in the FEL light pulse energy. Such a conversion process of the beam energy can be strongly affected by how the charged beam is distributed in the longitudinal phase space. This has a direct influence on the beam energy spread, which is a parameter proportional to the standard deviation of the beam energy distribution around the mean value. Moreover, the efficiency of such a process degrades as a function of the quality of the beam energy spread and is sensitive to all the factors concurring to its

*Electronic address: gianluca.orlandi@roma2.infn.it degradation. Therefore, the characteristics of the particular accelerator driving the FEL source are crucial for the spectral and the temporal distributions of the laser pulse.

Indeed, on linac driven FELs, the output laser efficiency is limited only by the peak current that is stored in the bunch and by the beam repetition rate. As a consequence, a linac based FEL can achieve a very high average power-above $1 \mathrm{~kW}$ - as has been demonstrated at Jefferson Lab [1] and at JAERI [2].

In a storage ring (SR) FEL the average kinetic energy of the beam is restored at each passage in the radiofrequency (rf) cavity, but the turn-by-turn energy exchange with the laser pulse degrades the beam quality leading to an increase of the energy spread $\sigma_{\epsilon}$, the socalled bunch heating $[3,4]$. The beam heating and the consequent bunch lengthening reduce the laser gain that is proportional to the charged beam density and to the inverse of the energy spread. The beam laser heating thus concurs itself to a degradation of the efficiency of the laser amplification process. This grows up to reach a saturation limit which may be quantitatively estimated by imposing the condition that the gain value is equal to the cavity losses. Consequently, in a storage ring driven FEL, the intracavity power is limited by the maximum value of the energy spread at the FEL equilibrium [5]. Finally, the useful laser power emerging from the optical cavity is a fraction of the intracavity power depending on the total absorption and on the output coupling of the optical resonator itself. 
Taking advantage of the pulse to pulse natural synchronization with the synchrotron radiation light for pump-probe two-color experiments, user applications of SRFELs demand a careful control of the laser output power [6-8]. At Super-ACO a useful average laser power of about $200 \mathrm{~mW}$ can be reached at $350 \mathrm{~nm}$ for beam current of about $80 \mathrm{~mA}$ [9]. Recently, in the UVSOR SRFEL, the extracted average laser power at $570 \mathrm{~nm}$ reached a maximum value of about $1 \mathrm{~W}$ for a beam current of $500 \mathrm{~mA}$ [8]. In the ELETTRA SRFEL, an extracted average power of more than $300 \mathrm{~mW}$ at $250 \mathrm{~nm}$ can be achieved for beam current of about $40 \mathrm{~mA}$ [10]. At the OK-4/Duke SRFEL, an extracted average power higher than $200 \mathrm{~mW}$ at about $225 \mathrm{~nm}$ can be obtained for a beam current of about $16 \mathrm{~mA}$ [11]. In the VUV OK-5/ Duke SRFEL, a power of about $10 \mathrm{~W}$ for a beam average current of $160 \mathrm{~mA}$ is foreseen for user applications at a wavelength between 200 and $240 \mathrm{~nm}$ [12].

In an electron storage ring both the bunch length and the energy spread undergo an anomalous increase above a current threshold [13-15] because of the beamenvironment interaction, i.e., the interaction of the relativistic charged beam with the self-induced electromagnetic field in the vacuum chamber and in the $\mathrm{rf}$ devices. Such a collective phenomenon, usually referred to as microwave instability, evolves with the stored current and significantly influences the SRFEL dynamics [16]. It may manifest in the potential-well distortion [15], being dominant in a low current limit, and in a turbulent bunch lengthening, which, conversely, is predominant in the high current limit. Since the microwave instability directly affects the natural energy spread of the charged beam, both the bunch heating and the laser saturation processes are changed and must be considered in a most general framework including the mentioned collective effects.

In some cases it has been shown that the laser heating counteracts the microwave instability [11,17-19]. In the OK-4/Duke SRFEL [11], the analysis of the measured power is carried out on the basis of the Renieri limit approach and of the results of a numerical code that is based on a 3D exact model of the SRFEL. By supposing that the microwave instability is completely counteracted by the laser heating, such a numerical code allows the bunch shape at the laser equilibrium to be predicted. The measured laser power of the ELETTRA SRFEL has been compared with a model where the laser heating is in competition with the microwave instability [19].

The relative role that the laser heating and the microwave instability play in shaping the Super-ACO longitudinal beam dynamics at the laser equilibrium is explored here. The measured laser power is compared with either the theoretical estimation of the Renieri limit or the numerical results of a macroparticle tracking code including laser pulse propagation and the effect of a longitudinal broad-band impedance model [20]. Preliminarily, the microwave instability effects on the Super-ACO beam longitudinal dynamics $[16,21]$ were studied by means of the Boussard model, which allows the turbulent bunch lengthening to be described. The laser induced effects on the longitudinal beam dynamics are then analyzed in the framework of the initial laser gain.

\section{SUPER-ACO BEAM DYNAMICS CHARACTERIZATION}

Super-ACO is a $800 \mathrm{MeV}$ positron storage ring that is equipped with a main $100 \mathrm{MHz}$ rf cavity for beam energy restoring and with an additional $500 \mathrm{MHz}$ harmonic rf cavity [21,22]. It has been installed to improve the storage ring performances under the two-bunches FEL operation [23]. In fact, it compresses longitudinally the bunch length as a function of the voltage amplitude. At nearly zero current, the bunch shortening that is operated by the 5 th harmonic cavity $(h=5)$ can be estimated according to the following formula [24]:

$$
\frac{\sigma_{\tau}^{h}}{\sigma_{\tau}}=\frac{1}{\sqrt{1+h V_{h} / V}}
$$

where $\sigma_{\tau}$ is the bunch length, corresponding to the operation with the main $\mathrm{rf}$ cavity active only at a voltage amplitude of $V$, and $\sigma_{\tau}^{h}$ is the bunch length occurring when the harmonic rf cavity is also active at a voltage amplitude equal to $V_{h}$.

With the small signal gain of the laser being proportional to the charged beam density [3,25], the FEL performances take advantage of the harmonic rf cavity action. The laser oscillation onsets when the small signal gain is larger than the optical cavity losses. Thus a reduction of the bunch length allows the laser oscillation threshold to be pushed towards a lower beam current value with a related attainment of a larger value of the equilibrium laser power. Several machine operation points can be set for different voltages of the harmonic rf cavity. The main parameters of the Super-ACO storage ring are reported in Table I.

The measurement of the beam longitudinal characteristics can be performed at Super-ACO as described in the following. A double sweep streak camera (Hamamatsu C5680) allows the bunch length $\sigma_{\tau}$ to be measured via the detection of the temporal duration of the synchrotron light pulse emerging from the dipole that is located before the FEL straight section [26]. By using a charge-coupled device camera, the beam energy spread $\sigma_{\epsilon}$ can be estimated by detecting the horizontal dimension $\sigma_{x}$ of the synchrotron light spot emerging from a dispersive section of the beam trajectory in the dipole [27]. By the knowledge of the horizontal emittance $\epsilon_{x}$, the dispersion function $\eta_{x}$, and the beta function $\beta_{x}$ at the dipole, the energy spread $\sigma_{\epsilon}$ (see Table I) can be estimated by means of the following equation: 
TABLE I. Main Super-ACO storage ring characteristics. Horizontal and vertical emittances $\left(\epsilon_{x}, \epsilon_{y}\right)$ and beam transverse dimensions $\left(\sigma_{x}, \sigma_{y}\right)$ at the center of the optical klystron. At the dipole placed in front of the FEL straight section the values of the dispersion function $\eta_{x}$ and of the beta function $\beta_{x}$ are 0.364 and $1.528 \mathrm{~m}$, respectively. $f_{s}$ is the synchrotron frequency.

\begin{tabular}{|c|c|c|c|}
\hline$C$ circumference $(\mathrm{m})$ & 72 & & \\
\hline$E_{s}$ beam energy $(\mathrm{MeV})$ & 800 & & \\
\hline Momentum compaction factor $\alpha$ & $1.48 \times 10^{-2}$ & & \\
\hline Dipole bending radius $(\mathrm{m})$ & 1.7 & & \\
\hline$P_{s}$ synchrotron power $((W)(I=1 \mathrm{~mA})$ & 23.28 & & \\
\hline Revolution period (ns) & 240 & & \\
\hline$T_{0}$ bunch interspacing $(\mathrm{ns})$ & 120 & & \\
\hline rf cavity $(\mathrm{MHz})$ & 100 & 500 & 500 \\
\hline Voltage $(\mathrm{kV})$ & 170 & 90 & 120 \\
\hline$\epsilon_{x}, \epsilon_{y}(\mathrm{~nm} \times \mathrm{rad})$ & 28,30 & 28,30 & 28,30 \\
\hline$\sigma_{x}, \sigma_{y}(\mu \mathrm{m})$ & 390,385 & 390,385 & 390,385 \\
\hline$f_{s}(\mathrm{kHz})$ nom. (absence of laser) & 14.3 & 27.3 & 30.4 \\
\hline$f_{s}(\mathrm{kHZ})$ exp. (absence/presence of laser) & $13.8 / 12.6$ & $20.8 / 23.6$ & $22.4 / 24.2$ \\
\hline$\tau_{s}$ synch. damping time & $10 \mathrm{~ms}$ & & \\
\hline$\sigma_{\tau}^{0}(\mathrm{ps})$ natural bunch length nom./exp. & $85 / 83$ & $45 / 45$ & 41 (nom.) \\
\hline$\sigma_{\epsilon}^{0}$ nom & $5.6 \times 10^{-4}$ & $5.6 \times 10^{-4}$ & $5.6 \times 10^{-4}$ \\
\hline
\end{tabular}

$$
\sigma_{x}^{2}=\epsilon_{x} \beta_{x}+\eta_{x}^{2} \sigma_{\epsilon}^{2}
$$

\section{Beam longitudinal dynamics}

Several concomitant effects may affect the longitudinal dynamics of an electron beam circulating in a storage ring. The mean equilibrium kinetic energy of the charged beam is the result of a net balance between the energy that is lost during a machine turn and the one that is received by the charged beam for each passage in the rf accelerating cavity. If the mean energy of the beam is turn by turn restored, on the contrary its natural energy spread undergoes a degradation with a consequent lengthening of the bunch. Collective effects are responsible for such a degradation of the natural longitudinal characteristics of the beam $[14,15]$. Since the FEL dynamics at Super-ACO involves only two symmetrically spaced bunches, in this context the attention will be pointed out only to the collective effects dealing with the longitudinal single-bunch dynamics.

Collective effects may induce degradation of the beam energy spread via either a dissipation of the kinetic energy of the beam or a nondissipative (elastic) interaction mechanism. To the first category belongs the space-charge effect, which is due to the action of the electric field corresponding to a nonuniform beam longitudinal distribution on the single particle, and the wake field effect, which takes into account the feedback between the charged beam and the self-induced field in the surrounding environment. To the second category belongs, for example, the Touschek intrabeam scattering [28] which, even though it is governed by a Coulomb elastic interac- tion of the charged particles in the beam, is as well responsible for an increase of the beam energy spread. Such collective effects are naturally different for their nature and interaction mechanism; nevertheless they are all joined together by the common characteristic to produce a degradation of the beam longitudinal quality. Their action on the beam energy spread leads to an identical final result; therefore both the laser heating and the foregoing collective mechanisms can be studied as competitive perturbing effects of the beam longitudinal dynamics.

The longitudinal dynamics of a charged beam in a storage ring can be formally described in terms of an oscillator model [29], where the harmonic oscillation of the generic particle of the beam around the synchronous phase of the sinusoidal rf field is damped by the synchrotron radiation emission and stimulated by a forcing term $\left(V_{\text {c.e. }}\right)$ that takes into account the influence of the collective effects. In the longitudinal phase plane, the generic particle of the beam can be described in terms of the following variables:

$$
\epsilon=\frac{E_{s}-E}{E_{s}}, \quad z=c\left(t_{s}-t\right),
$$

where $\epsilon$ is the relative energy deviation and $z$ the position deviation of the particle with energy $E$ and longitudinal coordinate $c t$ with respect to the synchronous one. The equation of motion reproducing the turn-by-turn particle motion reads

$$
\epsilon_{n+1}=\epsilon_{n}+\frac{e \dot{V}_{\mathrm{rf}} z_{n}}{E_{s}}-\frac{2 T \epsilon_{n}}{\tau_{s}}+2 \sigma_{\epsilon}^{0} \sqrt{\frac{T}{\tau_{s}}} r+V_{\text {c.e. }}\left(z_{n}\right),
$$




$$
z_{n+1}=z_{n}-\alpha C \epsilon_{n+1},
$$

where (see also Table I) $n$ refers to the number of machine turns, $\dot{V}_{\text {rf }}$ is the slope of the rf potential, $T$ is the machine revolution period, $\tau_{s}$ is the machine damping time, and $r$ is a random number accounting for quantum fluctuation noise. It can be demonstrated [29] that, for a damped harmonic oscillator model of the beam dynamics, the equilibrium energy spread can be related to the bunch length via the following equation:

$$
\sigma_{\tau}=\frac{\alpha}{2 \pi f_{s}} \sigma_{\epsilon}
$$

where $\alpha$ is the momentum compaction factor and $f_{s}$ the synchrotron frequency.

Here are considered only the collectives effects able to compete with the laser heating. In particular, the reciprocal interplay between the laser heating and the Touschek intrabeam scattering has been already treated in [30]. Moreover, no particular attention will be devoted to the space-charge effect whose action is only relevant for a low beam energy. Therefore the attention will be focused only on the so-called microwave instability effects. Under such a denomination, the collective effects due to the beam wake field induction on the vacuum chamber are usually indicated. Since the coupling between the charged beam and the induced wake field can be described in terms of an appropriate equivalent circuit, the effects of the microwave instability on the charged beam can be interpreted in terms of a suitable impedance. Depending on the characteristics of the surrounding environment, the circuital coupling between the charged beam and the induced field can be formally expressed in terms of an impedance containing a dominant resistive, inductive, or capacitive contribution. Therefore, resistive energy losses of the beam are due to the nonperfect conductor wall of the vacuum chamber. Narrow-band high $Q$-factor impedance can take into account the beam energy dissipated because of the excitation of resonant frequencies in the rf cavity (beam loading effect). Finally, a broad-band impedance, characterized by a low $Q$ factor, allows the wake field effects due to the transverse asymmetries and the dielectric discontinuities of the vacuum chamber to be described.

Microwave instability effects, typically affecting the single-bunch longitudinal dynamics in a storage ring, are the potential-well distortion and the turbulent bunch lengthening. The potential-well distortion results from an alteration of the rf cavity field, which is a beam induced excitation of the fundamental frequency in the cavity. It produces a modification of the longitudinal synchrotron oscillation regime of the beam which manifests in a synchrotron frequency shift and in a related scaling of the bunch length [15]. Such a synchrotron frequency shift scales as the cube of the bunch length, being thus predominant at low current.
At higher current a marked bunch lengthening regime occurs in a storage ring. Such an anomalous bunch lengthening is accompanied by a related increase of the energy spread. It can be interpreted in terms of a broad-band impedance coupling the charged beam to the induced wake field and described in terms of the Boussard model $[14,15,31]$. Being dominant at high current and with a strength increasing with the beam average current, the anomalous bunch lengthening may clearly play a competitive role with the laser heating. Among the microwave instability effects, the turbulent bunch lengthening is treated and its interplay with the laser heating is the object of the present investigation.

Under the hypothesis that the bunch length $\sigma_{\tau}$ and the energy spread $\sigma_{\epsilon}$ are linked [29] by a proportionality relation [Eq. (6)], both the turbulent anomalous energy spread and the bunch lengthening can be expressedversus the beam current-in terms of the following Boussard scaling formulas: [15]:

$$
\sigma_{\epsilon}=\sigma_{\epsilon}^{0}\left(\frac{I}{I_{\mathrm{th}}}\right)^{1 / 3}, \quad \sigma_{\tau}=\frac{\alpha \sigma_{\epsilon}^{0}}{2 \pi f_{s}}\left(\frac{I}{I_{\mathrm{th}}}\right)^{1 / 3},
$$

where $\sigma_{\epsilon}^{0}$, the so-called zero current value, is the energy spread value measured in the proximity of the zero current value and $I_{\text {th }}$ is the Boussard threshold current, which depends on the estimated broad-band impedance of the storage ring vacuum chamber.

The energy spread $\sigma_{\epsilon}$ and the bunch length $\sigma_{\tau}$ have been measured versus the average stored current for the following voltage amplitude $\left(V_{h}\right)$ values of the harmonic rf cavity: $V_{h}=0,90$, and $120 \mathrm{kV}$. The energy spread and the bunch length, measured for the only active main $\mathrm{rf}$ cavity $\left(V_{h}=0\right)$, are reported in Figs. 1(a) and 1(b). The measured values of $\sigma_{\epsilon}$ and $\sigma_{\tau}$ at low current in the proximity of the zero point $\left(\sigma_{\epsilon}^{0}\right.$ and $\left.\sigma_{\tau}^{0}\right)$ are compatible with the nominal ones. Both the bunch length and the energy spread increase as a function of the current with a similar behavior. When the additional harmonic cavity is powered at an amplitude voltage of $90 \mathrm{kV}$, the measured zero current values of the energy spread and of the bunch length are consistent with the nominal ones [Figs. 1(c) and 1(d) and Table I]. Moreover, both $\sigma_{\epsilon}$ and $\sigma_{\tau}$ increase smoothly versus the stored current with a similar shape. In particular, the expected behavior of the beam longitudinal dynamics under operations with the harmonic rf cavity is confirmed. The behavior of the energy spread versus current remains similar whatever the harmonic rf cavity voltage, while the measured bunch length undergoes the expected shortening. The estimated scaling factor of the bunch length at zero current is 0.54 , which is in good agreement with the theoretical one 0.52 , calculated via Eq. (1). For $V_{h}=120 \mathrm{kV}$, the behavior of $\sigma_{\epsilon}$ and $\sigma_{\tau}$ [Figs. 1(e) and 1(f)] confirms the modifications of the longitudinal beam dynamics in the presence of the harmonic rf cavity. The low current value of the energy 

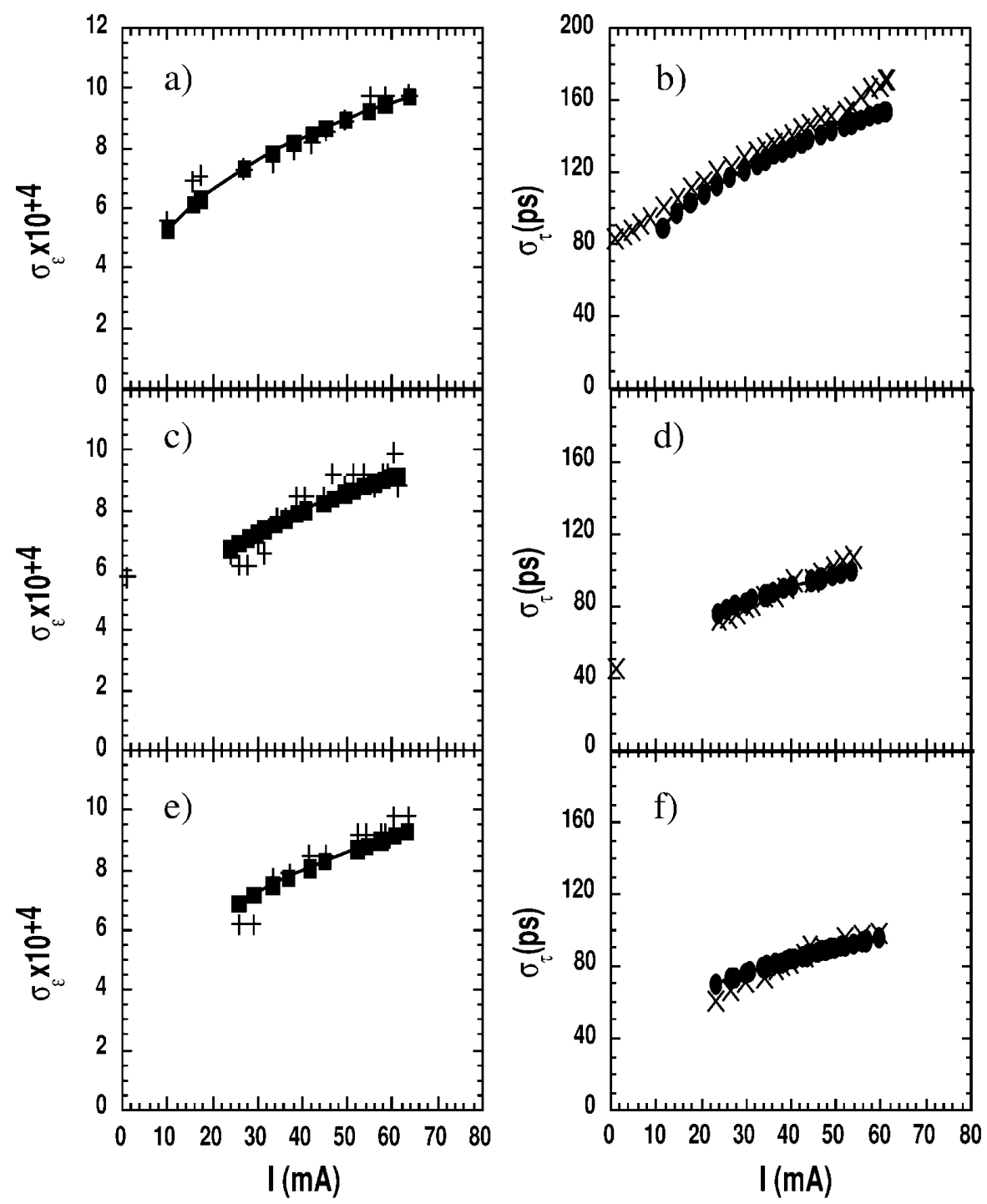

FIG. 1. Beam longitudinal dynamics characterization in the absence of a laser. Measured energy spread $\sigma_{\epsilon}(+)$ and Boussard limit estimation ( $\boldsymbol{\square}$ ) for (a) $V_{h}=0 \mathrm{kV}, \sigma_{\epsilon}^{0}=5.6 \times 10^{-4}$, and $I_{\mathrm{th}}=11.7 \mathrm{~mA}$; (c) $V_{h}=90 \mathrm{kV}, \sigma_{\epsilon}^{0}=5.6 \times 10^{-4}$, and $I_{\text {th }}=13.1 \mathrm{~mA}$; (e) $V_{h}=120 \mathrm{kV}, \sigma_{\epsilon}^{0}=5.6 \times 10^{-4}$, and $I_{\mathrm{th}}=13.1 \mathrm{~mA}$. Measured bunch length $\sigma_{\tau}(\times)$ and Boussard limit estimation $(\mathbf{O})$ for (b) $V_{h}=0 \mathrm{kV}, \sigma_{\tau}^{0}=83 \mathrm{ps}$, and $I_{\mathrm{th}}=11.7 \mathrm{~mA}$; (d) $V_{h}=90 \mathrm{kV}, \sigma_{\tau}^{0}=45 \mathrm{ps}$, and $I_{\mathrm{th}}=13.1 \mathrm{~mA}$; (f) $V_{h}=120 \mathrm{kV}, \sigma_{\tau}^{0}=41 \mathrm{ps}$, and $I_{\mathrm{th}}=13.1 \mathrm{~mA}$.

spread fits the nominal one; the behavior of the energy spread versus current is quite insensitive to the action of the harmonic cavity; the shortening of the bunch length is also confirmed by the experimental value $(0.49)$ of the scaling factor that is very close to the expected one (0.47).

For the different operating points of the Super-ACO storage ring, the increase of both the energy spread and the bunch length with current can be explained in terms of the microwave instability. Preliminarily, the existence of a proportionality relation linking the measured bunch length and the energy spread-whatever the average beam current - was checked for the different operating points. The applicability of Eq. (6) is confirmed by the observed linearity coupling the measured $\sigma_{\epsilon}$ and $\sigma_{\tau}$, as it is reported in Fig. 2. Therefore, the experimental value of the synchrotron frequency $f_{s}$ can be estimated (see Table I) via Eq. (6). The ratio of the synchrotron frequencies corresponding to an active or a passive harmonic $\mathrm{rf}$ cavity, respectively $f_{s, h}$ and $f_{s}$, may be estimated [32] via the following relation:

$$
\frac{f_{s, h}}{f_{s}}=\sqrt{1+h V_{h} / V} .
$$

A value of 1.08 for the experimental ratio is in agreement with the expected one.

Thanks to the observed linearity occurring between the measured $\sigma_{\epsilon}$ and $\sigma_{\tau}$, the microwave instability analysis of the measured bunch length and energy spread can be performed in terms of Eq. (7), as reported in Fig. 1. By 


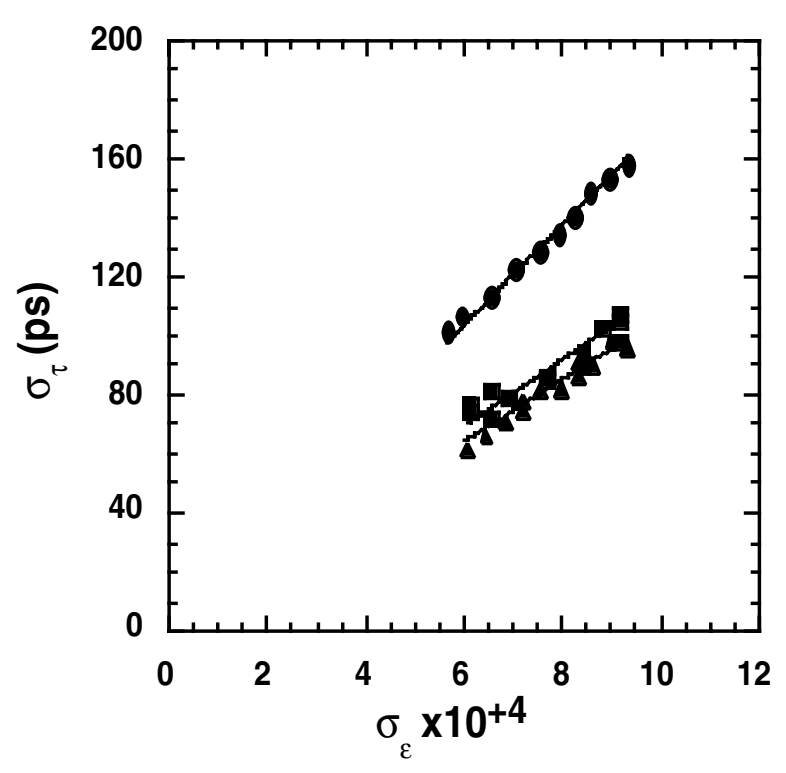

FIG. 2. Bunch length $\sigma_{\tau}(+)$ versus energy spread $\sigma_{\epsilon}$, both measured in the absence of a laser: $(\mathbf{O})$ for $V_{h}=0 \mathrm{kV}$, (ם) for $V_{h}=90 \mathrm{kV}$, and $(\boldsymbol{\Delta})$ for $V_{h}=120 \mathrm{kV}$.

fitting the measured energy spread versus the current, an estimation of $11.7 \mathrm{~mA}$ for the threshold current $I_{\text {th }}$ has been retrieved for $V_{h}=0 \mathrm{kV}$ [Fig. 1(a)]. When the harmonic rf cavity is active, a value of $13.1 \mathrm{~mA}$ for $I_{\text {th }}$ has been obtained [Figs. 1(c) and 1(e)]. These values are consistent with the ones which have already been presented in previous works [16], where a value for $I_{\text {th }}$ of $11 \mathrm{~mA}$ was retrieved for an estimated broad-band impedance of $4.3 \Omega$.

For the different operating points that have been considered, the measured energy spread and bunch length are affected by the microwave instability and can be correctly interpreted in terms of the Boussard scaling formulas. In the following, the competitive interplay of such an effect with another perturbating factor-the laser heating — will be studied.

\section{FEL SMALL SIGNAL GAIN}

The Super-ACO FEL is equipped with an optical klystron $[21,33]$ which is composed of two identical undulators with an intermediate dispersive section. At the resonance wavelength of the optical klystron, the coherent synchrotron radiation, partially stored in an optical resonator, interacts turn by turn with the charged beam leading to the laser amplification. The laser dynamics depends on the synchronization between the light pulse bouncing in the optical cavity and the charged beam circulating in the ring. The laser amplification, initially governed by the small signal gain $G_{0}$ [3], depends on the characteristics of the charged beam at the laser onset:

$$
G_{0} \propto \rho_{e} F_{f}\left(\sigma_{x}, \sigma_{y}\right) f\left(\sigma_{\epsilon}\right)
$$

TABLE II. Main Super-ACO FEL characteristics.

\begin{tabular}{lc}
\hline \hline Optical cavity length $(\mathrm{m})$ & 18 \\
Number of stored bunches & 2 \\
Undulator deflection parameter $K$ & 4.96 \\
Undulator spatial period $\lambda_{0}(\mathrm{~cm})$ & 12.9 \\
$N+N_{D}$ & 115 \\
Optimized modulation factor $f\left(f_{0}=0.88\right)$ & 0.53 \\
Filling Factor $F_{f}$ & 0.6 \\
Laser waist $w_{0}(\mu \mathrm{m})$ & 505 \\
Fundamental wavelength $\lambda(\mathrm{nm})$ & 350 \\
Wavelength tunability $(\mathrm{nm})$ & 10 \\
Spectral width $\Delta \lambda(\mathrm{FWHM})(\mathrm{nm})$ & 0.07 \\
\hline \hline
\end{tabular}

where $\rho_{e}$ is the beam spatial density and $F_{f}$ is the filling factor, representing the transverse overlapping between the laser mode and the charged beam with transverse sizes $\sigma_{x}$ and $\sigma_{y}$ [34]. The modulation rate $f$ [35] expresses the fringes modulation of the spectral intensity which results from the interference of the spontaneously emitted radiation emerging from the two undulators of the optical klystron. It depends on the beam energy spread $\sigma_{\epsilon}$ according to

$$
f=f_{0} e^{-8 \pi^{2}\left(N+N_{D}\right)^{2} \sigma_{\epsilon}^{2}}
$$

where $N$ is the number of undulator periods and $N_{D}$ the equivalent number of periods of the dispersive section. Table II reports the main parameters characterizing the Super-ACO FEL. The small signal gain $G_{0}$, plotted in Fig. 3 as a function of the average stored current, has been calculated by measuring the bunch characteristics for the following operating points: with a passive harmonic $\mathrm{rf}$ cavity and with the harmonic rf cavity active at a voltage amplitude of 90 and $120 \mathrm{kV}$. With respect to the passive case, higher gains can be achieved when the harmonic rf cavity is active because of the bunch shortening.

\section{LASER INDUCED BEAM HEATING}

The energy exchange occurring during the FEL oscillation between the laser pulse and the relativistic charged beam leads to an increase of the natural energy spread of the beam [3]. This is the effect of the laser heating and it can be formally treated by including, in the oscillator model for the beam longitudinal dynamics [Eq. (4)], a suitable additional forcing term, which is competitive with the one describing the microwave instability effect. From the analysis of the measured laser induced beam lengthening it should be possible to infer the reciprocal interplay occurring between such two perturbing effects. According to the theoretical model describing such a competitive mechanism [19,36], the absence in the experimental data of the expected laser induced bunch lengthening may be interpreted as the signature of a 


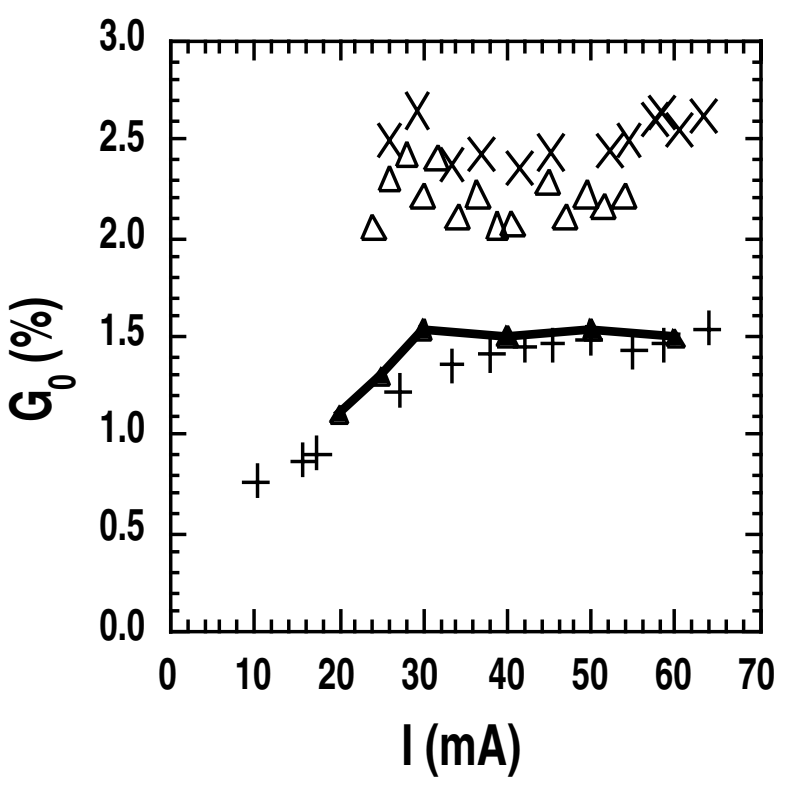

FIG. 3. Initial gain $G_{0}$ versus current. Experimental data (+) and related $(\boldsymbol{\Delta})$ numerical results computed by the STOK-2D code for $V_{h}=0 \mathrm{kV}$. Experimental data $(\triangle)$ and $(\times)$ for $V_{h}=$ $90 \mathrm{kV}$ and $V_{h}=120 \mathrm{kV}$, respectively.

dominant role of the laser heating with respect to the microwave instability.

The experimental characterization of the beam longitudinal dynamics under the laser heating has been carried out for the laser steady state at the same machine operating points that have been already treated in the subsection of II. In Figs. 4(a) and 4(b) the case of a passive harmonic rf cavity is reported, while the cases for a harmonic cavity that is powered at an amplitude voltage of 90 and $120 \mathrm{kV}$ are, respectively, described in Figs. 4(c)4(f). An increase of the energy spread and of the bunch length due to the FEL interaction has been observed at Super-ACO. The laser induced bunch lengthening is as more appreciable as higher is the average beam current. For $V_{h}=0 \mathrm{kV}$, the measured bunch length vs current [Fig. 4(b)] shows a relative increase up to $5 \%$ with respect to the reported data of Fig. 1(b), while the measured energy spread [Fig. 4(a)] does not show substantial variation [see Fig. 1(a)]. For $V_{h}=90 \mathrm{kV}$, the maximum relative enhancement of the energy spread [Fig. 4(c)] and of the bunch length [Fig. 4(d)] are, respectively, 6\% and $12 \%$. Conversely, for $V_{h}=120 \mathrm{kV}$, the bunch length variation [Fig. 4(f)] maintains within the measurement error ( $2 \mathrm{ps})$ and the relative increase of the energy spread [Fig. 4(e)] reaches a maximum value of $10 \%$.

As it results from such an analysis, the observed laser heating of the bunch is relatively small and weaker than what is expected by estimating how much the laser gain has to decrease in order to reach the mirror losses value, typically of the order of $1 \%$. For example, for $V_{h}=0 \mathrm{kV}$, if the whole laser power were dissipated in a simple beam heating process, the beam energy spread at the laser equilibrium should experience, with respect to the measured values in the absence of a laser, a relative increase ranging from $32 \%$ to $50 \%$ for a beam current between 30 and $60 \mathrm{~mA}$. For $V_{h}=90$ and $120 \mathrm{kV}$, the beam energy spread enhancement should be between $45 \%$ and $60 \%$ for the beam current ranging from 30 to $50 \mathrm{~mA}$. The discrepancy between the observed effect, due to the laser heating, and the expected one has been already quantitatively interpreted [36] in terms of a phenomenological model joining the laser heating and the microwave instability in the longitudinal beam dynamics. The model showed that the action of such two phenomena contributes to an equivalent increase of the energy spread. According to it, the discrepancy between the observed and the expected values could be attributed to the reciprocal interplay of the laser heating and of the microwave instability. The laser heating drives the longitudinal beam dynamics towards the equilibrium state after a preliminary annihilating process of the original imprinting due to the microwave instability.

The experimental characterization of the beam longitudinal dynamics in the presence of the laser has been completed with the analysis of the reciprocal dependence of the measured bunch length $\sigma_{\tau, O N}$ and energy spread $\sigma_{\epsilon, O N}$. The behavior of $\sigma_{\tau, O N}$ vs $\sigma_{\epsilon, O N}$ (Fig. 5) confirms the existence of a proportionality relation which has been already observed in the absence of the laser. Because of the laser heating, the longitudinal beam dynamics is anyway modified. Indeed, the proportionality factor between $\sigma_{\tau, O N}$ and $\sigma_{\epsilon, O N}$ changes, leading to a different estimation of the synchrotron frequencies, which are reported in Table I. Finally, a ratio equal to 1.03 of the estimated synchrotron frequencies for $V_{h}=90 \mathrm{kV}$ and $V_{h}=$ $120 \mathrm{kV}$ has been retrieved via Eq. (8). Such a value varies slightly with respect to the analogous one which has been obtained in the absence of a laser.

In conclusion, the longitudinal beam dynamics is affected by the laser heating: the induced beam lengthening is less important than the expected one, but the longitudinal synchrotron oscillation regime is modified.

\section{FEL OPERATIONS AND MEASURED POWER ANALYSIS}

The results of the previous section clearly indicate that the interplay between the microwave instability and the laser heating is governed by a complex mechanism. According to the previously reported data analysis, the laser heating process leading to the laser equilibrium state can be interpreted as the result of a partial damping effect of the microwave instability in the longitudinal beam dynamics. The study of such a competitive interplay and the quantitative evaluation of the degree of such a suppression can be achieved by analyzing the equilibrium laser power. In fact, it has been demonstrated that, in a 

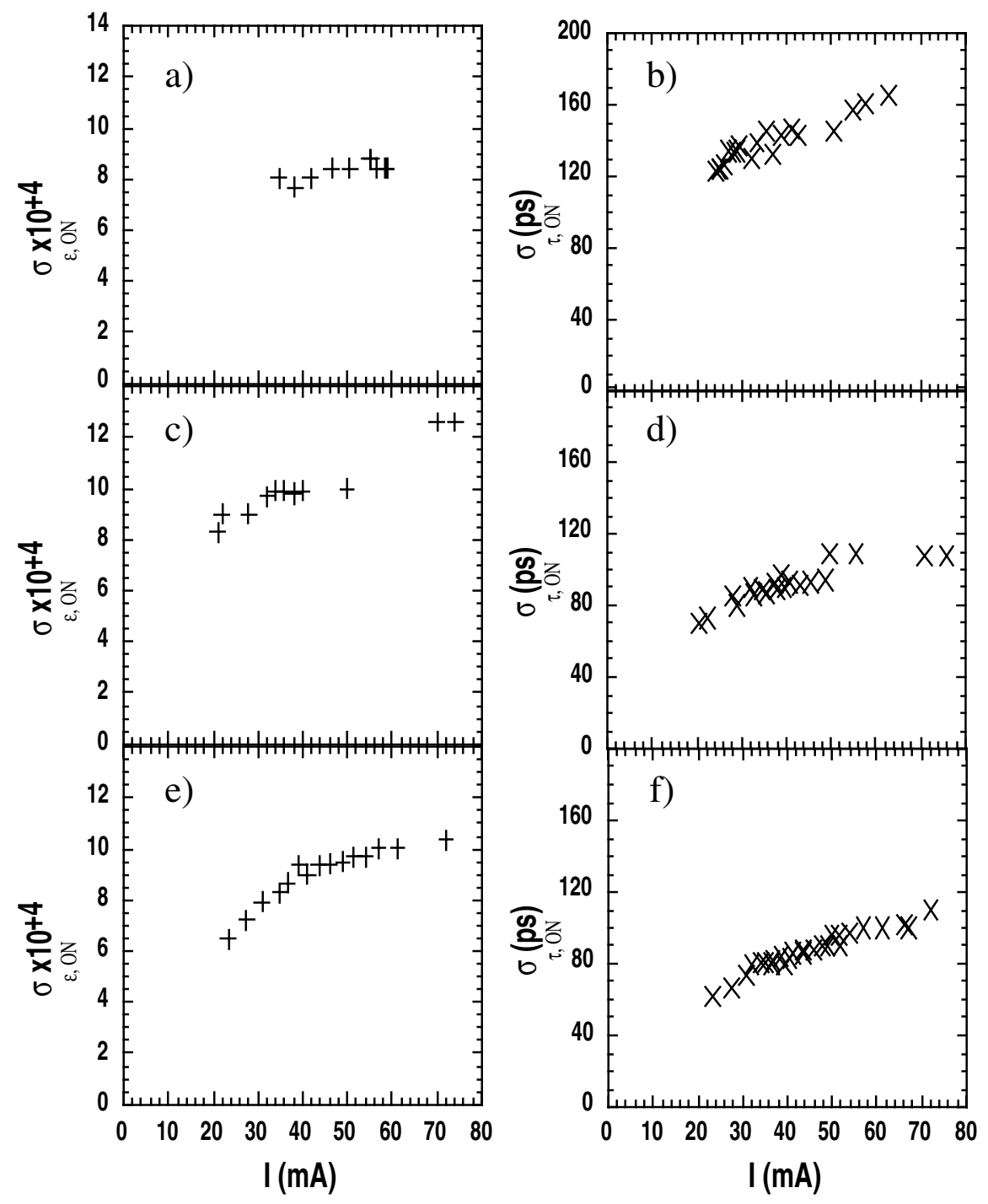

FIG. 4. Beam longitudinal dynamics characterization under laser heating. Measured energy spread $\sigma_{\epsilon}(+)$ for (a) $V_{h}=0 \mathrm{kV}$; (c) $V_{h}=90 \mathrm{kV}$; (e) $V_{h}=120 \mathrm{kV}$. Measured bunch length $\sigma_{\tau}(\times)$ for (b) $V_{h}=0 \mathrm{kV}$; (d) $V_{h}=90 \mathrm{kV}$; (f) $V_{h}=120 \mathrm{kV}$.

storage ring FEL, the laser average output power is proportional to an efficiency function which increases towards a limit value for a decreasing value of the initial energy spread of the beam [4]. From the analysis of the laser power, useful information about the evolution of the beam energy spread - from the initial to the laser steady state - can be retrieved.

Measurements of the equilibrium FEL power have been carried out at Super-ACO by detecting, by means of an optical power meter (Newport, model 835), the laser light which is extracted from the rear mirror of the optical cavity. The equilibrium FEL power, measured versus the stored beam current, has been also analyzed according to the Renieri limit [5]. By measuring the beam energy spread, in the presence $\left(\sigma_{\epsilon, O N}\right)$ and in the absence $\left(\sigma_{\epsilon}\right)$ of the laser, and the total losses $L$ of the optical cavity, the FEL average power can be estimated according to

$$
P_{L}=\eta_{C} 8 \pi\left(N+N_{D}\right) f P_{S}\left(\sigma_{\epsilon, O N}^{2}-\sigma_{\epsilon}^{2}\right),
$$

where the efficiency $\eta_{C}$ is defined as $\eta_{C}=T / L$. T is the mirror transmission and $P_{S}$ is the synchrotron power that is emitted per revolution by the average current circulating in the storage ring (Table I). The mirror losses $L$ of the Super-ACO optical cavity can be estimated by measuring the exponential envelope of the extinguishing light pulse that is stored in the optical cavity. This is obtained by varying the revolution time of the electron bunch with respect to the round-trip period of the laser pulse bouncing in the optical cavity. For the Super-ACO FEL the temporal synchronization is suitably set by varying the frequency of the main rf cavity. The detuning of the main cavity frequency $\mathrm{rf}$-by means of a frequency shift $\Delta f_{\text {rf }}$-allows the losses to be evaluated according to

$$
L=\frac{\Delta f_{\mathrm{rf}}}{f_{\mathrm{rf}}} \frac{T_{0}}{\tau}
$$




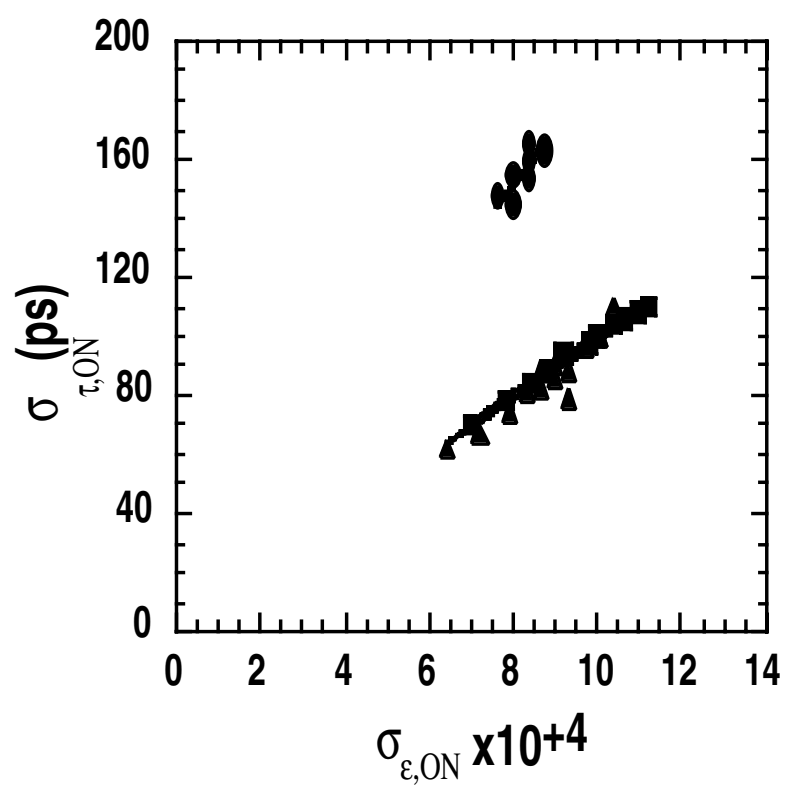

FIG. 5. Bunch length (+) versus energy spread both measured under laser heating: $(\mathbf{O})$ for $V_{h}=0 \mathrm{kV} ;(\boldsymbol{\square})$ for $V_{h}=90 \mathrm{kV}$ and (A) for $V_{h}=120 \mathrm{kV}$.

where $T_{0}$ is the time interval between the two positron bunches stored in the ring and $\tau$ is the time constant of the exponential envelope [37]. The extinguishing light pulse in the optical cavity is detected by means of a stroboscopic technique with a dissector [38].

For different operating points of the accelerator, the equilibrium laser power has been measured versus the stored current. In Fig. 6(a) the measured data for a passive harmonic rf cavity are reported. In Figs. 6(b) and 6(c) the acquired data for an active harmonic rf cavity - respectively at 90 and $120 \mathrm{kV}$ - are shown as well. Each series of experimental data manifests a quite regular increase as a function of the stored beam current. Moreover, by a comparison of the three data series, the laser power grows up as the voltage amplitude of the harmonic rf cavity is raised from the zero value to the reference values of 90 and $120 \mathrm{kV}$, according to what is expected because of the bunch shortening.

In order to study the interplay between the FEL heating and the microwave instability, the laser power has been analyzed $[18,19]$ via Eq. (11) in the two limit cases which are able to occur. In the first case, it is supposed that the laser heating does not produce an alteration of the initial microwave instability imprinting the bunch characteristics in the absence of the laser. Therefore, Eq. (11) is implemented by referring to the beam characteristics which are measured on the condition of laser equilibrium and the absence of the laser at the same beam current value. In the second case, the laser heating is conversely supposed to be able to completely suppress the initial microwave instability. Thus, the equilibrium laser power is estimated as a function of the beam current, according

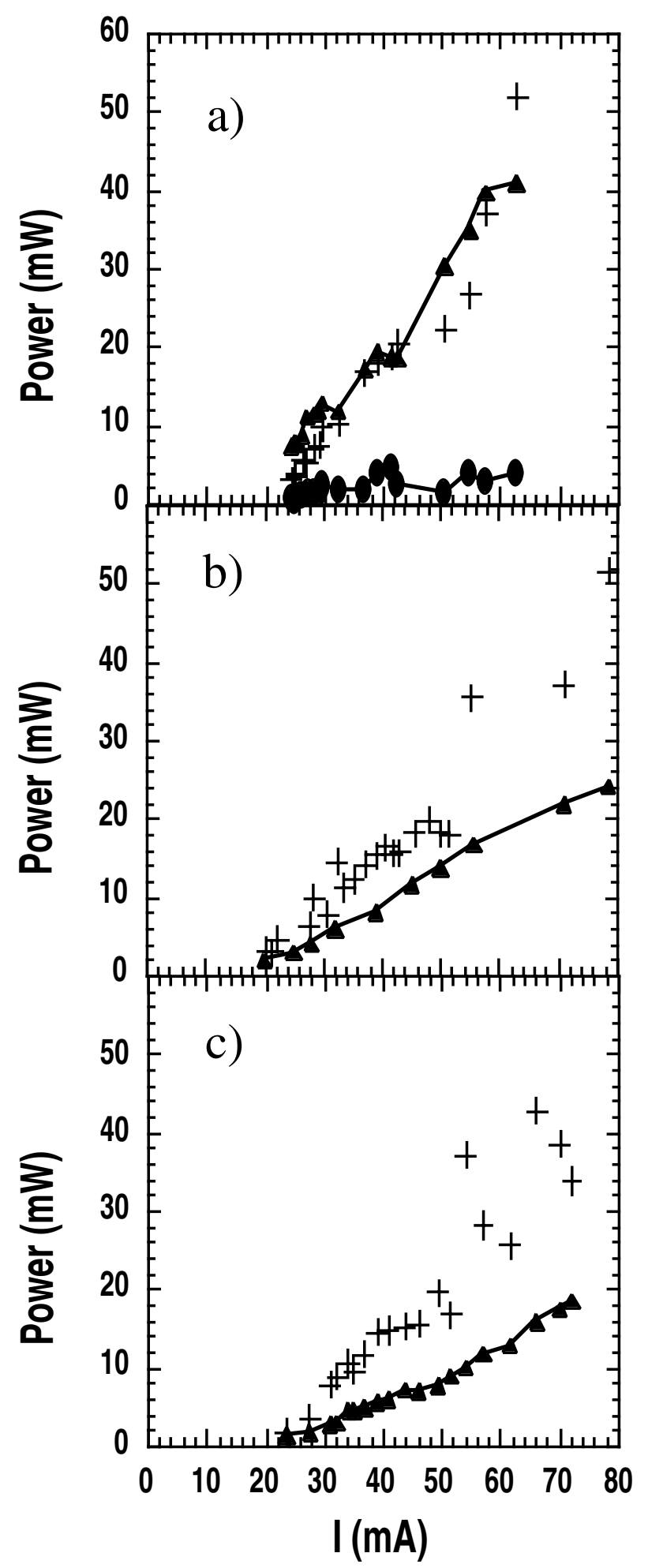

FIG. 6. Equilibrium laser power measurements (+) vs current and Renieri limit analysis performed by comparing the bunch length measured under laser heating either (O) with the corresponding value measured in the absence of a laser or (A) with the zero current value. (a) $V_{h}=0 \mathrm{kV}, T=$ $3 \times 10^{-4}, L=1 \%$, and $\sigma_{\tau}^{0}=83 \mathrm{ps;} \mathrm{(b)} V_{h}=90 \mathrm{kV}, T=$ $1.7 \times 10^{-4}, \quad L=1 \%$, and $\sigma_{\tau}^{0}=45 \mathrm{ps} ;$ (c) $V_{h}=120 \mathrm{kV}$, $T=1.7 \times 10^{-4}, L=1.13 \%$, and $\sigma_{\tau}^{0}=41 \mathrm{ps}$. 
to the Renieri limit, by comparing the bunch characteristics, measured at the laser equilibrium, and those measured at the so-called zero current value. Moreover, thanks to the proportionality relation between the measured bunch length and the energy spread for the different considered experimental cases, the theoretical limit [Eq. (11)] is calculated by referring to either the one or the other quantity.

For the case of a passive harmonic rf cavity [Fig. 6(a)] a best fit of the experimental data can be obtained when the Renieri limit is calculated by referring to the zero current value for the quantity that is measured in the absence of the laser. In such a case, the analysis of the measured laser power clearly indicates that the longitudinal beam dynamics reaches an equilibrium state which is characterized by a complete suppression of the microwave instability due to the laser heating effect.

Similarly, sets of experimental data, acquired with the harmonic rf cavity active at voltage amplitudes of $90 \mathrm{kV}$ [Fig. 6(b)] and $120 \mathrm{kV}$ [Fig. 6(c)], have been analyzed. For both cases, the Renieri limit has been calculated by comparing the quantities, measured at the laser equilibrium, with the zero current ones. In such a case as well the analysis of the measured laser power confirms the conclusion that has been drawn from the analysis of the passive harmonic rf cavity data.

In conclusion, the analysis of the laser power in the framework of the Renieri limit clearly indicates that the beam longitudinal dynamics at the laser equilibrium is the result of a counteraction of the microwave instability due to the laser heating. The relative role of the different perturbing sources of the beam longitudinal dynamics has also been analyzed at the laser equilibrium in terms of the results of a suitable numerical code as reported in the following.

\section{Numerical analysis of SuperACO FEL output power}

A study of the effect of the microwave instability on the FEL performances has been carried out with the numerical code STOK-2D that couples the longitudinal electron dynamics in the storage ring with the laser pulses propagation [20]. The numerical code computes the longitudinal beam dynamics in the SRFEL by treating the beam as an ensemble of macroparticles whose motion is governed by a damped harmonic oscillation equation with a forcing term. It can be formally expressed by an equation identical to Eq. (4) provided that a suitable forcing term is added:

$$
\epsilon_{n+1}=A+V_{\mathrm{FEL}}\left(\epsilon_{n}, z_{n}\right)
$$

where $A$ is the right member of Eq. (4) and $V_{\mathrm{FEL}}\left(\epsilon_{n}, z_{n}\right)$ takes into account the laser heating. The evolution equations of the code take into account the effect of a linearized $\mathrm{rf}$ potential, the radiation damping, the associated quantum noise, the presence of a broad-band impedance, and the laser pulse. The effect of the longitudinal impedance is modeled by an energy kick due to the longitudinal wake field of a RLC (resistive-inductive-capacitive) resonator [14]. Finally, the effect of the FEL is modeled by an energy kick that takes into account the effect of energy spread widening and energy losses due to the interaction of the beam with the radiation field [39].

In the evolution equations, the FEL energy kick contribution $V_{\mathrm{FEL}}\left(\epsilon_{n}\right)$ is composed of two terms describing the energy spread widening and the energy loss due to the FEL emission, respectively. The former one is given by

$$
\begin{aligned}
V_{\mathrm{FEL}}\left(\epsilon_{n}, z_{n}\right)= & \frac{1}{N} \sqrt{\frac{T}{\tau_{s}} W\left(z_{n}\right)}\left(\frac{\sin \left[2 \pi N\left(\epsilon_{n}+\epsilon_{0}\right)\right]}{2 \pi N\left(\epsilon_{n}+\epsilon_{0}\right)}\right) \\
& \times \sin \left[\phi-2 \pi N\left(\epsilon_{n}+\epsilon_{0}\right)\right],
\end{aligned}
$$

where $\phi$ is a random phase, $N$ is the number of undulator periods, $\epsilon_{0}$ is the relative deviation of the energy of the synchronous particle with respect to the optical klystron resonant energy, $W(z)=0.6\left(\tau_{s} / T\right)\left(I(z) / I_{S}\right)$ with $I(z)$ the FEL intracavity power density and $I_{S}$ the intracavity saturation intensity [20]. The latter one is computed according to Madey's theorem [3] as the derivative

$$
V_{\mathrm{FEL}}\left(\epsilon_{n}, z_{n}\right)=\frac{1}{2} \frac{d}{d \epsilon_{0}}\left[V_{\mathrm{FEL}}\left(\epsilon_{n}, z_{n}\right)\right]^{2} .
$$

The effects of the wake field $V_{\text {c.e. }}\left(z_{n}\right)$ [Eq. (4)] are described via a broad-band resonator RLC model. This can be formally written as

$$
V_{\text {c.e. }}\left(z_{n}\right)=-\frac{e^{2}}{E_{S}} \sum_{k} N_{k} K\left(z_{n}-z_{k}\right),
$$

where the term $K(z)$ describes the broad-band resonator RLC model [20]:

$$
K(z)=\frac{\omega_{0} R}{Q} e^{w_{0} z / 2 Q c}\left(\cos \left(\omega_{1} z / c\right)+\frac{\sin \left(\omega_{1} z / c\right)}{\sqrt{4 Q^{2}-1}}\right)
$$

with $z<0, w_{0}=k_{0} / \sigma_{\tau}$, and $\omega_{1}=\omega_{0} \sqrt{1-1 / 4 Q^{2}}(Q$, resonator quality factor).

In the code the parameters of the RLC impedance model are appropriately chosen to reproduce the SuperACO bunch lengthening and the energy spread curves as a function of current. The numerically estimated gain curve vs the beam current is reported in Fig. 3 for operation with the only active main rf cavity and compared with the analogous one which have been obtained according to Eq. (9) from the measured beam characteristics. It is clearly seen that the FEL gain does not increase at large current owing to the combined effect of the anomalous bunch lengthening and energy spread due to the RLC impedance. Moreover, using for the cavity losses a value of $L=1 \%$, the laser threshold of $15 \mathrm{~mA}$ is also well reproduced. 


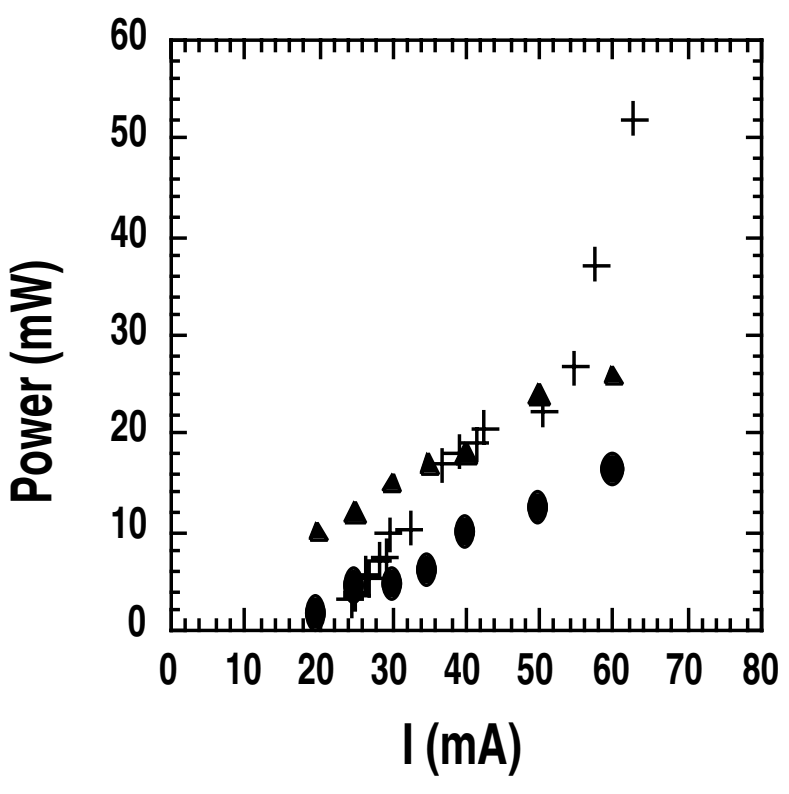

FIG. 7. Output FEL power results for the $100 \mathrm{MHz}$ rf cavity only active, obtained by the STOK-2D numerical code supposing a $L=1 \%$ and $T=3 \times 10^{-4}$. The numerical results obtained with $(\mathbf{O})$ and without $(\boldsymbol{\Delta})$ inclusion of the microwave instability in the numerical code are compared with the experimental data (+) already reported in Fig. 6.

In order to check the hypothesis of the existence of a competitive interplay between the laser heating and the microwave instability in the achievement of the steady state regime for the Super-ACO longitudinal beam dynamics at the FEL equilibrium, several numerical simulations of the laser power have been performed for the case of the only active $100 \mathrm{MHz}$ rf cavity.

Two limit cases have been considered: in the first case the laser terms coexist with the microwave instability perturbation $\left[V_{\text {c.e. }}\left(z_{n}\right) \neq 0\right]$; in the second one the microwave instability is switched off $\left[V_{\text {c.e. }}\left(z_{n}\right)=0\right]$. The results, shown in Fig. 7, are compared with the experimental data which are also reported in Fig. 6(a). In the low current regime (below $30 \mathrm{~mA}$ ) the measured power is fitted by the curve corresponding to the case $V_{\text {c.e. }}\left(z_{n}\right) \neq 0$. In the high current regime (between 35 and $50 \mathrm{~mA})$ a quite good agreement is shown between the measured power and the numerical curve corresponding to $V_{\text {c.e. }}\left(z_{n}\right)=0$. This behavior indicates that the relative weight of the laser heating with respect to the microwave instability increases with the current: at low current the laser heating starts as a weak perturbation to reach a dominant role at higher current. The continuous transition of the power from one limit case to the other one at the intermediate current regime describes the increasing weight of the laser heating. The analysis of the FEL power that has been obtained via the numerical code [20] confirms the conclusions already drawn on the basis of the Renieri limit interpretation. They indicate that the longitudinal beam dynamics in the Super-ACO storage ring is indeed the result of a competitive equilibrium between laser heating and microwave instability with a dominating role of the first one.

\section{CONCLUSION}

Several sets of the equilibrium laser power measured in the Super-ACO SRFEL for different operating conditions have been analyzed and presented. The longitudinal beam dynamics characterization, based on the measured bunch length and energy spread vs the beam current, is clearly interpreted in terms of a microwave instability model. The broad-band impedance model seems adequate to give a realistic description of the beam dynamics of the Super-ACO storage ring. On the basis of the Renieri limit analysis and the numerical results of a macroparticle tracking code at the laser equilibrium, it turns out that the Super-ACO beam longitudinal dynamics is dominated by the laser heating, being otherwise weakly influenced by the microwave instability. This latter plays a primary role even in a 3 rd generation electron storage ring. In a storage ring FEL the microwave instability operates in the same current range where the laser is active. Therefore, a correct study of the laser dynamics in a storage ring FEL cannot avoid a careful analysis of its interplay with the microwave instability.

\section{ACKNOWLEDGMENTS}

This work was supported by the European Community TMR Contract No. ERBFMGECT980102. The support of the Super-ACO machine staff is particularly acknowledged.

[1] G. R. Neil, C. L. Bohn, S.V. Benson, G. Biallas, D. Douglas, H. F. Dylla, R. Evans, J. Fugitt, A. Grippo, J. Gubeli, R. Hill, K. Jordan, R. Li, L. Merminga, P. Piot, J. Preble, M. Shinn, T. Siggins, R. Walker, and B. Yunn, Phys. Rev. Lett. 84, 662 (2000).

[2] E. J. Minehara, M. Sawamura, R. Nagai, N. Kikuzawa, M. Sugimoto, R. Hajima, T. Shizuma, T. Yamauchi, and N. Nishimori, Nucl. Instrum. Methods Phys. Res., Sect. A 445, 183 (2000); E. J. Minehara, Nucl. Instrum. Methods Phys. Res., Sect. A 483, 8 (2002).

[3] J. M. J. Madey, Nuovo Cimento Soc. Ital. Fis. 50B, 64 (1979).

[4] G. Dattoli and A. Renieri, Nuovo Cimento Soc. Ital. Fis. 59B, 1 (1980).

[5] A. Renieri, Nuovo Cimento Soc. Ital. Fis. 53B, 160 (1979).

[6] M. E. Couprie, P. Tauc, F. Merola, A. Delboulbé, D. Garzella, T. Hara, and M. Billardon, Rev. Sci. Instrum. 65, 1485 (1994).

[7] M. Marsi, M. E. Couprie, L. Nahon, D. Garzella, T. Hara, R. Bakker, M. Billardon, A. Delboulbé, G. Indlekofer, and A. Taleb-Ibrahimi, Appl. Phys. Lett. 70, 895 (1995). 
[8] M. Hosaka, S. Koda, M. Katoh,J. Yamazaki, K. Hayashi, K. Takashima, T. Gejo, and H. Hama, Nucl. Instrum. Methods Phys. Res., Sect. A 483, 146 (2002).

[9] T. Hara, M. E. Couprie, D. Garzella, L. Nahon, M. Marsi, R. Bakker, and M. Billardon, J. Electron Spectrosc. Relat. Phenom. 80, 317 (1996).

[10] M. Trovò, J. A. Clarke, M. E. Couprie, G. Dattoli, D. Garzella, A. Gatto, L. Giannessi, S. Günster, N. Kaiser, M. Marsi, M.W. Poole, D. Ristau, and R. P. Walker, Nucl. Instrum. Methods Phys. Res., Sect. A 483, 157 (2002).

[11] V. N. Litvinenko, S. H. Park, I.V. Pinayev, and Y. Wu, Nucl. Instrum. Methods Phys. Res., Sect. A 475, 65 (2001).

[12] V. N. Litvinenko, O. A. Shevchenko, and N. A. Vinokurov, Nucl. Instrum. Methods Phys. Res., Sect. A 475, 97 (2001).

[13] J. L. Laclare, CERN Report No. 85-19, 1985, p. 377.

[14] A. Chao, Physics of Collective Beam Instabilities in High Energy Accelerators (John Wiley and Sons, New York, 1983).

[15] H. Wiedemann, Particle Accelerator Physics (Springer, New York, 1998), Vol. II.

[16] G. Dattoli, L. Mezi, A. Renieri, M. Migliorati, M. E. Couprie, D. Garzella, D. Nutarelli, C. Thomas, G. De Ninno, and R. P. Walker, Nucl. Instrum. Methods Phys. Res., Sect. A 471, 403 (2001).

[17] M. E. Couprie and P. Elleaume, Nucl. Instrum. Methods Phys. Res., Sect. A 259, 77 (1987).

[18] R. Roux and M. Billardon, Nuovo Cimento Soc. Ital. Fis. 122A, 513 (1999).

[19] R. Bartolini, G. Dattoli, L. Giannessi, M. Marsi, L. Mezi, M. Migliorati, M. Trovò, and R. P. Walker, Nucl. Instrum. Methods Phys. Res., Sect. A 491, 507 (2002).

[20] R. Bartolini, G. Dattoli, L. Giannessi, and L. Mezi, Nucl. Instrum. Methods Phys. Res., Sect. A 492, 276 (2002); R. Bartolini, "STOK-2D: A Computer Program for the Analysis of the Longitudinal Beam Dynamic in a Storage Ring Optical Klystron FEL," ENEA internal report, Frascati, Italy.

[21] R. Roux, M. E. Couprie, R. J. Bakker, D. Garzella, D. Nutarelli, L. Nahon, and M. Billardon, Phys. Rev. E 58, 6584 (1998).

[22] M. E. Couprie, C. Bazin, M. Bergher, M. Billardon, P. Elleaume, J. M. Ortega, Y. Petroff, R. Prazeres, and M. Velghe, Nucl. Instrum. Methods Phys. Res., Sect. A 282, 455 (1989).
[23] M. E. Couprie, D. Nutarelli, R. Roux, L. Nahon, B. Visentin, A. Delboulbé, G. Flynn, and M. Billardon, Nucl. Instrum. Methods Phys. Res., Sect. A 407, 215 (1998).

[24] G. Flynn, J. M. Godefroy, J. Polian, F. Ribeiro, and T. Ruan, in Proceedings of the 6th EPAC, Stockholm, Sweden, 1998 (IOP, Bristol, UK, 1998), pp. 1767-1769.

[25] J. M. J. Madey, J. Appl. Phys. 42, 1906 (1971).

[26] R. Roux, M. E. Couprie, T. Hara, R. J. Bakker, B. Visentin, M. Billardon, and J. Roux, Nucl. Instrum. Methods Phys. Res., Sect. A 393, 33 (1997).

[27] R. J. Bakker, M. E. Couprie, L. Nahon, D. Nutarelli, R. Roux, A. Delboulbé, D. Garzella, A. Nadji, B. Visentin, and M. Billardon, in Proceedings of the 5th EPAC, Sitges, Spain, 1996 (IOP, Bristol, UK, 1996), pp. 667-669; M. Hosaka, H. Hama, J. Yamazaki, and T. Kinoshita, Nucl. Instrum. Methods Phys. Res., Sect. A 407, 234 (1998).

[28] J. Le Duff, in Proceedings of the CAS CERN Accelerator School 95-06, 1995, Vol. II.

[29] M. Sands, SLAC Report No. 121 UC-28, 1970.

[30] R. Bartolini, G. Dattoli, L. Giannessi, L. Mezi, A. Renieri, C. Bruni, M. E. Couprie, D. Garzella, G. L. Orlandi, and M. Migliorati, Phys. Rev. E (to be published).

[31] D. Boussard, CERN Report No. CERN/LABII/RF/INT/ 75-2, 1975.

[32] M. Billardon, M. E. Couprie, D. Nutarelli, G. Flynn, P. Marin, R. Roux, and M. Sommer, in Proceedings of the 6th EPAC, Stockholm, Sweden, 1998 (Ref. [24]), pp. 954-956.

[33] N. A. Vinokurov and A. N. Skrinsky, Preprint No. INP7788, Novosibirk, 1978.

[34] W. Colson and P. Elleaume, Appl. Phys. B 29, 101 (1982).

[35] P. Elleaume, J. Phys. Colloque 44, 333 (1983).

[36] G. Dattoli et al., Nucl. Instrum. Methods Phys. Res., Sect. A 393, 70 (1997); R. Bartolini et al., Phys. Rev. Lett. 87, 134801 (2001); R. Bartolini et al., Phys. Rev. E (to be published).

[37] D. Garzella, Ph.D. thesis, Université de Paris Sud, Paris, 1996.

[38] E. I. Zinine, Nucl. Instrum. Methods Phys. Res., Sect. A 208, 439 (1983); M. E. Couprie, V. M. Popik, E. I. Zinine, A. Delboulbé, D. Garzella, M. Velghe, and M. Billardon, Nucl. Instrum. Methods Phys. Res., Sect. A 318, 59 (1992).

[39] G. Dattoli et al., Free Electron Laser Theory and Related Topics (World Scientific, Singapore, 1993). 